\title{
A Systematic Review of Interventions for Inappropriate Sexual Behavior of Children and Adolescents with Developmental Disabilities
}

\author{
Laurie McLay $^{1}$ - Amarie Carnett ${ }^{2} \cdot$ Gaye Tyler-Merrick $^{1} \cdot$ Larah van der Meer $^{2}$
}

Received: 5 June 2015 / Accepted: 2 September 2015 / Published online: 21 September 2015

(C) Springer Science+Business Media New York 2015

\begin{abstract}
This review provides a systematic analysis of studies that evaluated interventions for inappropriate sexual behavior(s) of children and adolescents with developmental disabilities. Searches of databases, reference lists, and journals yielded 12 studies that met the predetermined inclusion criteria. Each study was summarized in terms of (a) participant characteristics, (b) dependent variables, (c) research design, (d) measures and data collection procedures, (e) independent variables, (f) treatment integrity, (g) results, and (h) level of certainty. All of the 12 studies reported decreases in the target behavior as the result of intervention. The most common intervention involved the use of multi-component behavioral strategies. Clinical implications and suggestions for future research are discussed.
\end{abstract}

Keywords Developmental disabilities · Autism . Inappropriate sexual behavior - Childhood masturbation . Children · Adolescents

\section{Introduction}

Historically, individuals with developmental disabilities have been regarded as uninterested in relationships, devoid of sexual urges, and/or sexually deviant. As a result, prior to the 1970 s, very little research was conducted into the sexuality

Laurie McLay

laurie.mclay@canterbury.ac.nz

1 School of Health Sciences, College of Education, University of Canterbury, Private Bag 4800, Christchurch 8140, New Zealand

2 School of Education, Victoria University of Wellington, Wellington, New Zealand of those with disabilities (Milligan and Neufeldt 2001). Childhood sexual development and the sexual behavior of young people with disabilities is an area of research that remains particularly neglected. According to Sandfort and Rademakers (2000), there are two key reasons for this. The first is due to the sensitive nature of this topic. Parents are often reluctant to acknowledge that their children engage in sexual behavior and are even more reluctant to allow their child to participate in research in this area. The second is related to methodology. Sexual behavior is generally viewed to be a private event, and for obvious ethical reasons, it is not appropriate to observe children or adolescents engaging in masturbation or other types of sexual behavior nor are we able to ask particular probing questions if children are used as the source of information in this area. A third potential consideration relates to the important cultural and religious factors that may restrict or prohibit research in this area.

Sexual experimentation and exploration fits within the normal spectrum of human sexual behavior in prepubescent years. In particular, masturbation behavior has been observed to occur in utero (Meizner 1987) and during early childhood (Friedrich et al. 1998). According to Leung et al. (1993), masturbation occurs throughout the lifespan and most commonly occurs at 4 years of age and then again during adolescence. When defining normal or abnormal sexual behavior, the social, cultural, and familial contexts, the setting in which the behavior is occurring, and developmental norms need to be considered. When masturbation behavior is conducted in a private setting and is not excessive in nature, it is considered to be a normal part of child development. This behavior, however, may be considered to be inappropriate when it occurs for a sustained period of time and/or in public spaces and thus may warrant the need for intervention.

It is generally reported that people with developmental disabilities demonstrate typical patterns of physical development 
during puberty. However, according to Sullivan and Caterino (2008), any emotional changes and/or the emergence of sexual urges can be delayed in children with autism spectrum disorder (ASD). The onset of puberty, and the emergence of sexual urges, can be problematic for children with developmental disabilities as this can coincide with an increase in challenging behavior at around the same age (Eaves and Ho 1996). As children with ASD are not always aware of, or do not respond to norms that dictate the social conventions regarding sexual behavior, inappropriate sexual behavior (ISB) can emerge.

Children with ASD in particular are reported to frequently engage in public masturbation (Ruble and Dalrymple 1993; Stokes and Kaur 2005), inappropriate touching of others (Clements and Zarowska 2000; Stokes and Kaur 2005), and fetishistic behavior (Ruble and Dalrymple 1993). There are several plausible explanations for this behavior. Firstly, sex education is not always provided to individuals with disabilities and is an area that is often ignored due to the potential embarrassment it may cause parents. Secondly, it is suggested that the predisposition of those with ASD, to engage in selfstimulatory behavior, may contribute towards repeated, inappropriate sexual behavior (Dalldorf 1983; Realmuto and Ruble 1999). Thirdly, unlike typically developing children, those with intellectual disabilities, particularly ASD, often require adapted and intensive instructional practices in order to acquire an understanding of the social and behavioral skills that might be learnt incidentally by others (Barnhill 2007; Gerhardt 2006; Hatton and Tector 2010). Due to the specific social deficits associated with ASD, these children and young people may have difficulty discriminating between public and private settings and may have impaired "theory of mind" (Baron-Cohen 2001). As a result, these children may have difficulty developing prosocial friendships and intimate relationships, may misunderstand relationship boundaries, may have difficulty understanding those behaviors that are appropriate in public versus private settings (Gougeon 2010; Hellemans et al. 2007), and may be excluded from social groups that provide typically developing children with peer feedback (Sullivan and Caterino 2008). Ludlow (1991) further suggests that the attention provided in response to the ISB of people with disabilities may provide strong social reinforcement for this behavior.

Premature sexual development, or precocious puberty, is another area that may pose challenges for children with developmental disabilities and their families. Precocious puberty is defined as the onset of signs of puberty prior to the age of 8 years in girls and 9 years of age in boys (Motzin 1993). It is thought that precocious puberty can be caused by damage to the central nervous system and may occur as a result of specific genetic disorders, such as Down syndrome and Fragile $\mathrm{X}$. It is also something that occurs more commonly among females than males. It is important to be aware of the increased risk of early onset puberty among those with developmental disability and, in treatment, to consider the possibility that neurobiological factors might be contributing towards the ISB.

There is little agreement in the literature regarding what can be considered ISB. For the purpose of this review, and based upon the literature on this subject, the term ISB is used to refer to those sexual behaviors that could be deemed to be excessive, obsessive, occur in public, violate others, are aggressive in nature, and/or imitate adult acts that are considered to be socially inappropriate.

When individuals engage in ISB, there are profound implications for their social and familial relationships, as well as their community and home life. In clinical practice, parents of children with disabilities will often voice concern about their child's ISB and may seek advice in this regard (Mallants and Casteels 2008). To date, there is limited research that has investigated different approaches to the treatment of childhood masturbation and even fewer studies that have examined ISB among children with ASD or other types of developmental disabilities. As a result, there is little consensus among clinicians regarding best practice for treatment of ISB, and the services and supports that are provided in this area are often limited.

Mallants and Casteels (2008) conducted a review of the literature which investigated assessment and treatment approaches for childhood masturbation among those with typical development. They developed recommended treatment guidelines as a product of this review. In these guidelines, it is suggested that the process should begin with an assessment that includes questions about the child's development, including affect, behavior, and sleep; questions about potential genitourinary or other medical problems; and consideration of cultural, familial, historical, relationship, and environmental variables. The signs of sexual abuse and/or skin irritation also need to be eliminated. In terms of treatment for childhood masturbation (CM), the authors highlight the fact that evidence-based treatment of early CM is lacking. When the assessment identifies that there are no additional confounding factors, it is recommended that treatment should focus on parent education and support. Educational processes should aim to alter parent perceptions of CM so that it is not viewed as pathology but, rather, a typical part of child development. It is further recommended that occurrences of $\mathrm{CM}$ should either be ignored or redirected and not punished due to the potential reinforcement that can come from responding to this behavior. Finally, the authors concluded that developmentally appropriate sex education should be provided to children in order to help them to understand what socially appropriate sexual behavior is and what it is not.

The only other identified review of treatments for ISB was conducted by Tarnai (2006). Tarnai (2006) conducted a review of 17 studies that investigated treatment for the socially inappropriate masturbation behavior of people with cognitive 
disabilities. Successful intervention outcomes were a criterion for inclusion in this review. The author of this review developed ten key guidelines for managing socially inappropriate masturbation in people with cognitive disabilities. These guidelines included (1) provide education related to appropriate socio-sexual skills; (2) avoid being overly tolerant of inappropriate autoerotic behavior; (3) avoid over-reacting to inappropriate autoerotic behaviors; (4) eliminate a medical or organic cause or the effects of medication on the masturbation behavior; (5) ensure that the interpersonal needs of individuals with disabilities are met; (6) teach people to have a positive attitude about their sexuality; (7) target self-regulation of masturbation behavior; (8) incorporate the use of positive support methods, including reinforcement procedures and sex education; (9) avoid the use of interventions that you do not have consent to implement; and (10) maintain procedures that ensure ethical treatment practices during therapy.

In addition to the procedures recommended by Tarnai (2006), there is further evidence in the literature to suggest that treatment for ISB among those with disabilities should include a focus on providing sex education, as well as individualized instructional strategies. For example, Koller (2000) suggests that sex education for individuals with ASD should include instruction that is brief, repetitive, and specific; inappropriate behaviors should be promptly responded to and redirected; individuals should be taught an appropriate setting and time during which they can engage in sexual behavior; and private time should be proactively scheduled.

In spite of the suggested value in providing sex education to children with disabilities, it is evident that we need to enhance our understanding of evidence-based practice in this area. Schaafsma et al. (2013) explored the development of sex education programs for people with intellectual disabilities and found that in general, these programs did not have clear outcome objectives, lacked theoretical underpinnings, had not been systematically evaluated, and the development of such programs did not involve consultation with relevant groups.

It is essential to understand the lifecourse trajectory of ISB. In early childhood and prepubescence, the occurrence of these behaviors can have a significant impact on the development of friendships, familial relationships, social participation, and the individual's inclusion in regular education settings. If ISB persists or escalates into adulthood, this behavior can further impact on the formation of social relationships, intimate relationships, home living, and community integration. If appropriate treatments cannot be found, these children could be at at-risk of sexual abuse, particularly given the increased vulnerability and risk for sexual violence that children with disabilities can face (Jones et al. 2012; Koller 2000).

This review aims to build on previous reviews of the literature by identifying and systematically evaluating treatments for ISB for children and adolescents with developmental disabilities. The clinical implications of the findings and potential future research directions will be discussed.

\section{Methods}

\section{Search Procedures}

A search of the electronic databases PsycINFO, Education Resources Information Centre (ERIC), Education Research Complete, and PubMed was conducted in March 2014 by both the first and second authors. The search terms "masturbation," "sexual behavior," and "genital stimulation" were individually combined with the terms "autism," "ASD," "disabilities," "developmental disabilities," and "intellectual disabilities." Entering a combination of the terms "autism" and "sexual behavior" into PubMed yielded the greatest number of results, with 122 articles. The combination of search terms "sexual behavior" and "intellectual disabilities" and "autism" and "sexual behavior" into PsycINFO resulted in 115 and 98 articles, respectively. This was the second and third most productive combination of search terms. In addition to the database searches, ancestry searches were conducted using the reference list of each article that was found. Finally, a search of Google Scholar was conducted using the aforementioned search terms in order to identify any literature that may have been overlooked. No additional articles were found as the result of searching Google Scholar or ancestry searching. Each of the articles identified as a result of the collective searches were examined in order to determine whether they met criteria for inclusion in this review.

The literature search was updated in May 2015 by the fourth author. This did not result in any additional articles but did reveal two potentially relevant papers (Early et al. 2012; Mallika et al. 2013) that were later excluded as they did not meet inclusion criteria.

\section{Inclusion and Exclusion Criteria}

Due to the paucity of research that has investigated treatments for inappropriate sexual behavior in children with disabilities, this review did not restrict consideration to studies that were double-blinded, placebo-controlled or within-participant experimental trials, and included case reports. Any empirical research studies that were identified and met the specified criteria were included in this review and no studies were excluded based upon whether or not treatments were demonstrated to be effective. Furthermore, given the limited amount of current research in this area, articles were included regardless of the year of publication.

Each study was evaluated using a predetermined inclusion criteria which included the following parameters: (a) the studies were required to have been published in academic, peer- 
reviewed journals and published in English and (b) the participants in the study included at least one child with a developmental disability who was 18 years of age or younger. This age was selected as the cutoff point as it is generally considered to be the age at which adolescence ends and adulthood begins; (c) the studies were required to have examined the efficacy of the treatment approach as a treatment for ISB; and (d) the studies were required to include outcome measures that examined the effect of treatment on ISB, including observation measures and standardized assessment tools. Based on the literature that exists on this subject, ISB included public masturbation, public displays of genitalia, and inappropriate interpersonal sexual acts (e.g., rubbing genitalia against another person). Treatment or intervention was defined as the implementation of a treatment approach or method in order to decrease ISB and/or teach an appropriate alternative to the sexual behavior. This included pharmacological treatments and also included treatments that were conducted in either a one-to-one or group therapy format.

\section{Data Extraction}

Twelve articles met the above inclusion criteria. The remaining articles were excluded as they did not include people with developmental disabilities, did not investigate treatments for ISB, or included participants who were older than 18 years of age. The 12 studies retained were evaluated and summarized according to the following criteria: (a) participants, (b) research design, (c) dependent variables, (d) independent variables, (e) measures and data collection, (f) treatment integrity, (g) results (including measures of generalization and maintenance), and (h) level of certainty.

For the purpose of this review, generalization of outcomes is defined based on the occurrence or non-occurrence of the target behavior under non-teaching conditions or when these teaching conditions are varied. This is derived from Stokes and Baer's (1977) definition.

The term treatment integrity is used to capture methods that were applied to ensure the intervention was implemented in the way in which it was intended. This includes recording inter-observer agreement (IOA) data, the provision of preintervention training for those implementing treatment, direct observation and recording of target behaviors, checklists to evaluate fidelity of implementation, and social validity checklists. Table 1 presents a summary of this information for each of the studies included in this review.

The criteria for evaluating the certainty of evidence described in this review are based upon the procedures outlined by Mulloy et al. (2010) and developed by Simeonson and Bailey (1991) and Smith (1981). Three levels of certainty are described in this study. These are suggestive, preponderant, and conclusive certainty (Simeonson and Bailey 1991; Smith 1981). Simeonson and Bailey (1991) define suggestive evidence as studies which produce outcomes that could be plausible. This generally refers to studies that have not utilized a well-accepted experimental design (e.g., a multiple baseline design or a randomized controlled trial), resulting in the lowest level of certainty. A preponderance of evidence describes those studies which conclude that any change is likely to be the result of the intervention or treatment that was implemented. The evidence, however, is able to be refuted. In this paper, as in the Mulloy et al. (2010) review, studies that were considered to provide a preponderant level of evidence included the following: (a) an experimental study design, (b) IOA and treatment fidelity data was gathered, as appropriate, (c) operational definitions of dependent variables were provided, as appropriate, (d) sufficient detail was provided in order to allow replication, and (e) their ability to control for alternative explanations of treatment effects was significantly limited.

Conclusive evidence describes those studies in which treatment outcomes are almost certainly true. Studies that provide conclusive evidence possess all of the methodological features of the studies that provide preponderant levels of evidence and also attempt to control for extraneous variables that may also account for treatment effects.

In alignment with the procedure outlined in the Mulloy et al. (2010) review, the studies in this paper were coded according to whether the effects of treatment were positive, negative, or mixed. A positive effect was used to describe those within-subject design studies where all participants had positive treatment outcomes or there were statistically significant differences between groups in the group design studies. Mixed effects refer to those outcomes in which some participants demonstrated positive treatment effects, while other participants did not, and/or positive outcomes were identified for some but not all dependent variables. Negative effects describe those studies in which no treatment effects were noted for any of the participants in a within-group design, or the difference between groups was not statistically significant for those studies that employed a between groups design.

\section{Interobserver Agreement and Reliability}

In total, 18 articles were identified as possibly meeting criteria for inclusion in this review. In order to ensure the accuracy of the database search procedure, the first and second authors reviewed each of the 18 studies against inclusion and exclusion criteria. This resulted in $100 \%$ agreement that 12 studies met review criteria. The remaining six articles were primarily excluded as they did not report on the treatment of inappropriate sexual behavior or they did not include individuals with developmental disabilities.

Each of the 12 studies were evaluated by the first author according to the eight criteria reported in Table 1. The second author later checked the accuracy of the data reported on in each study, by responding to the following questions: (a) is 


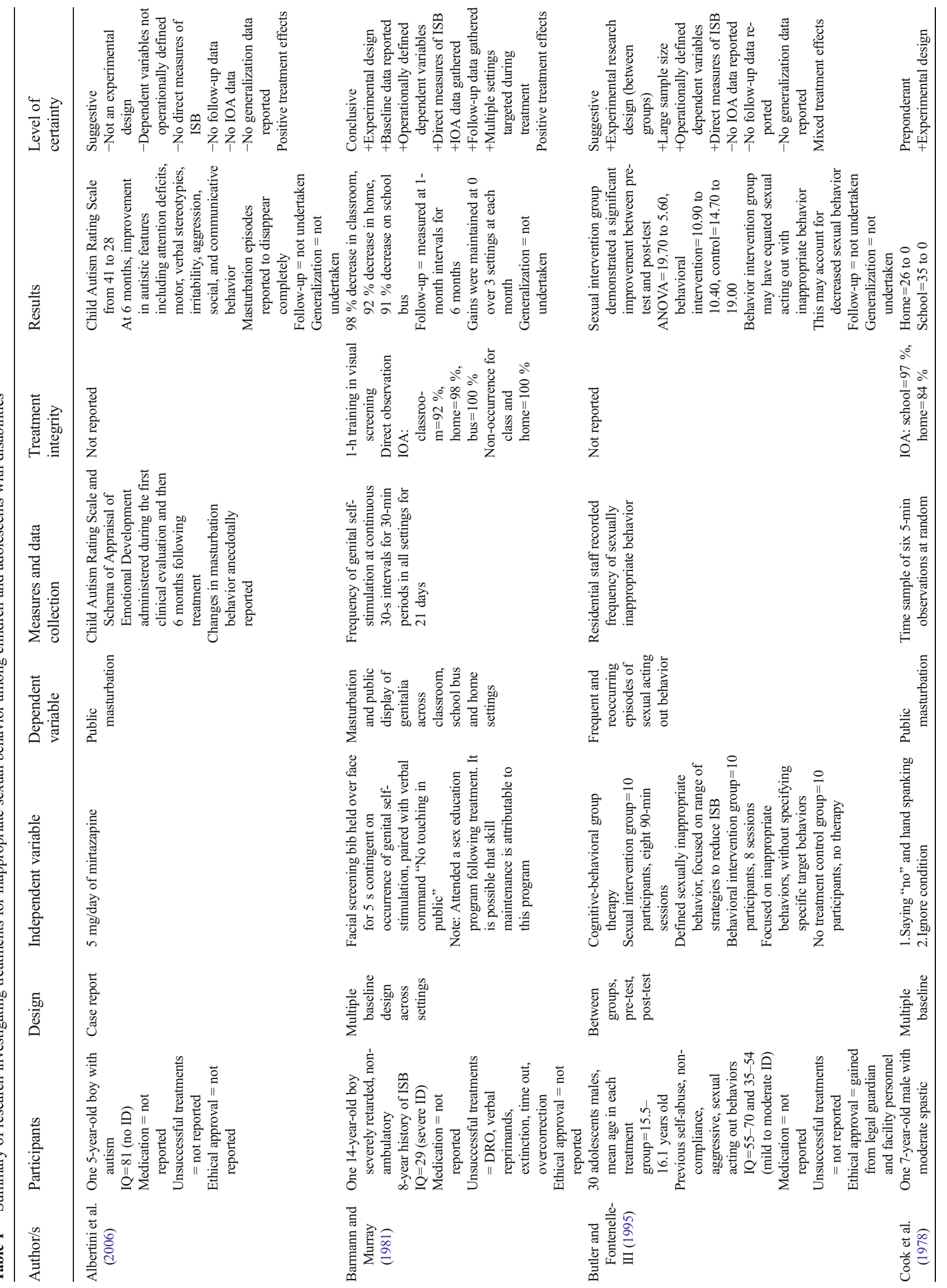




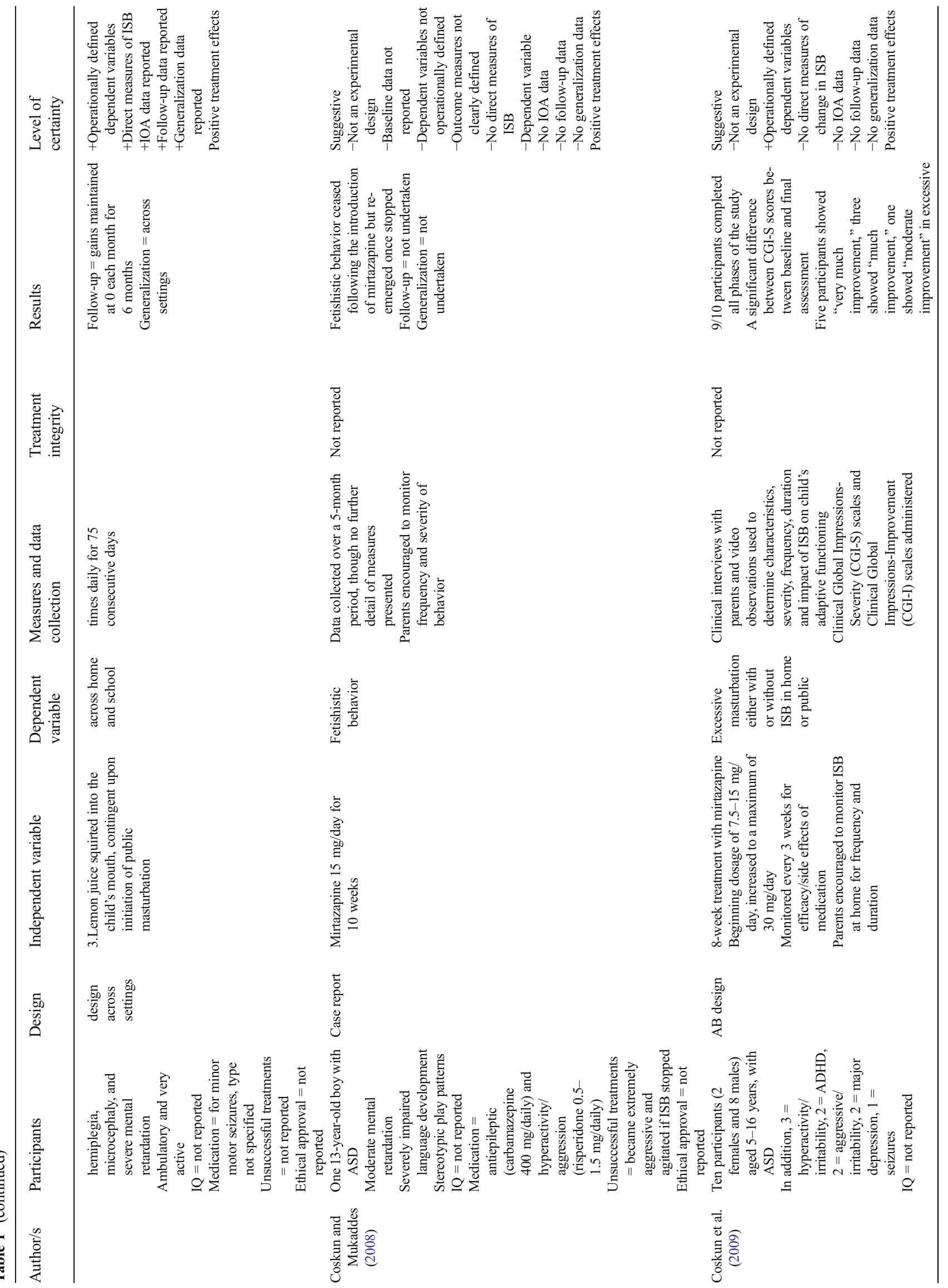




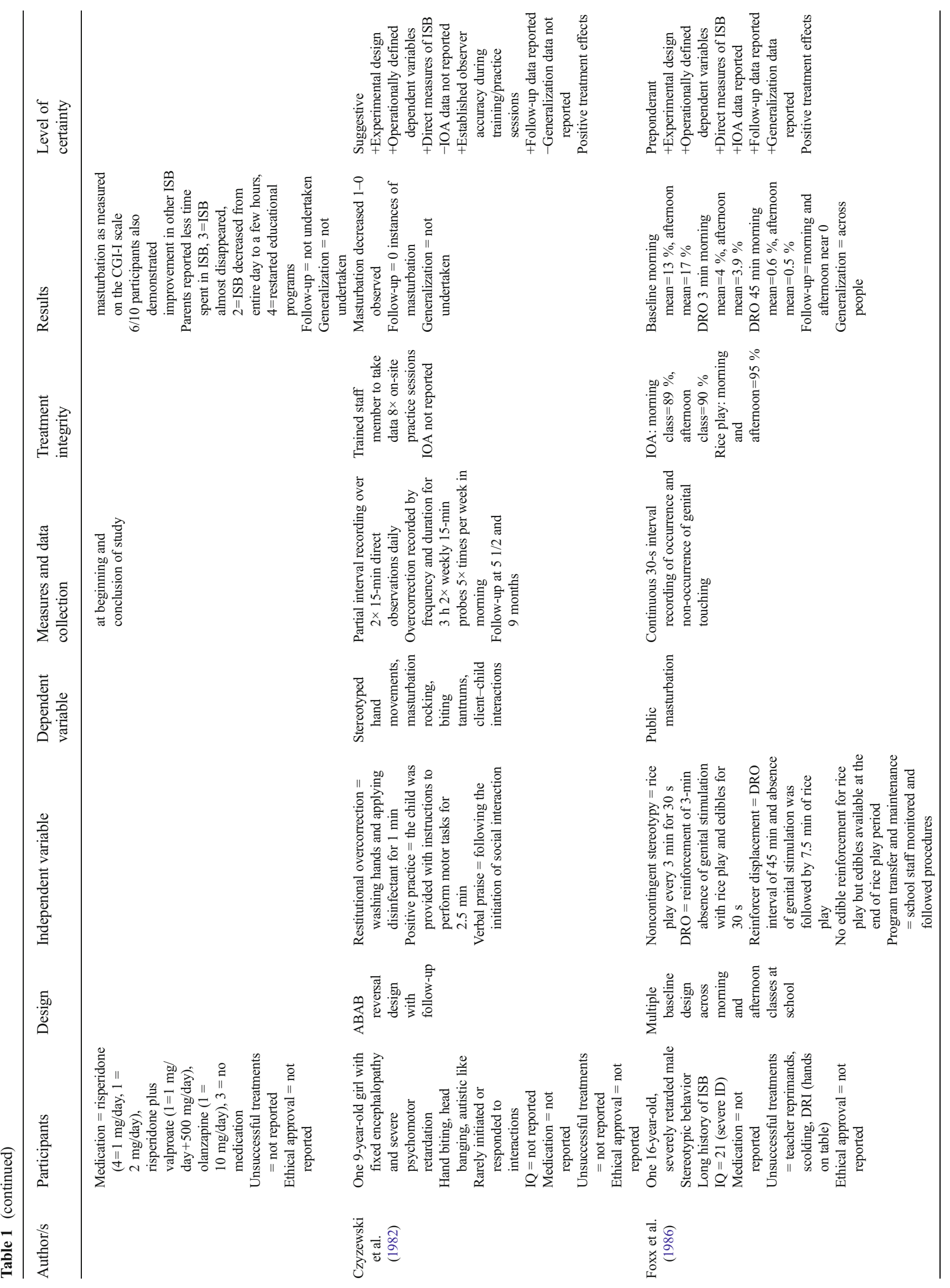




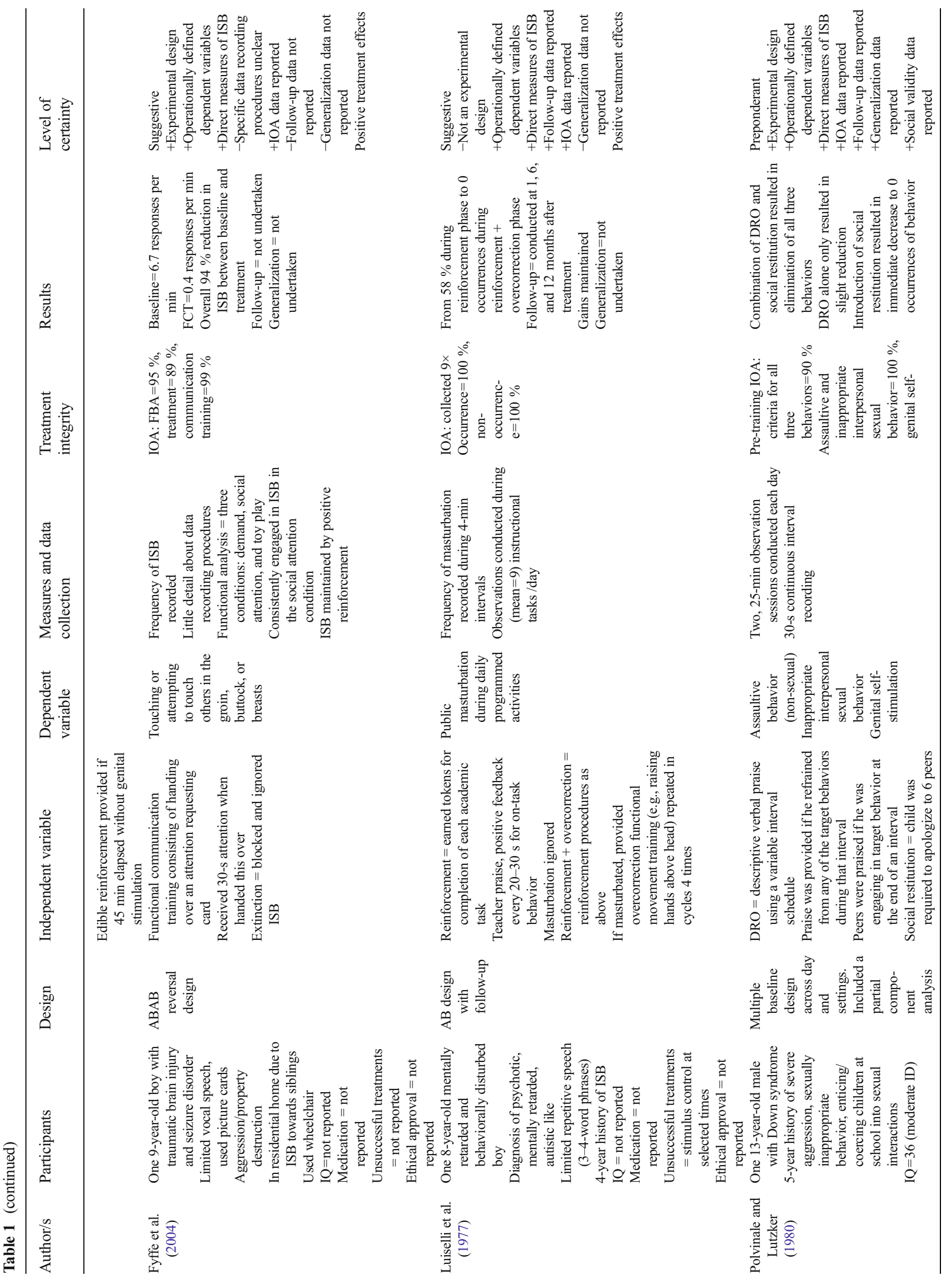




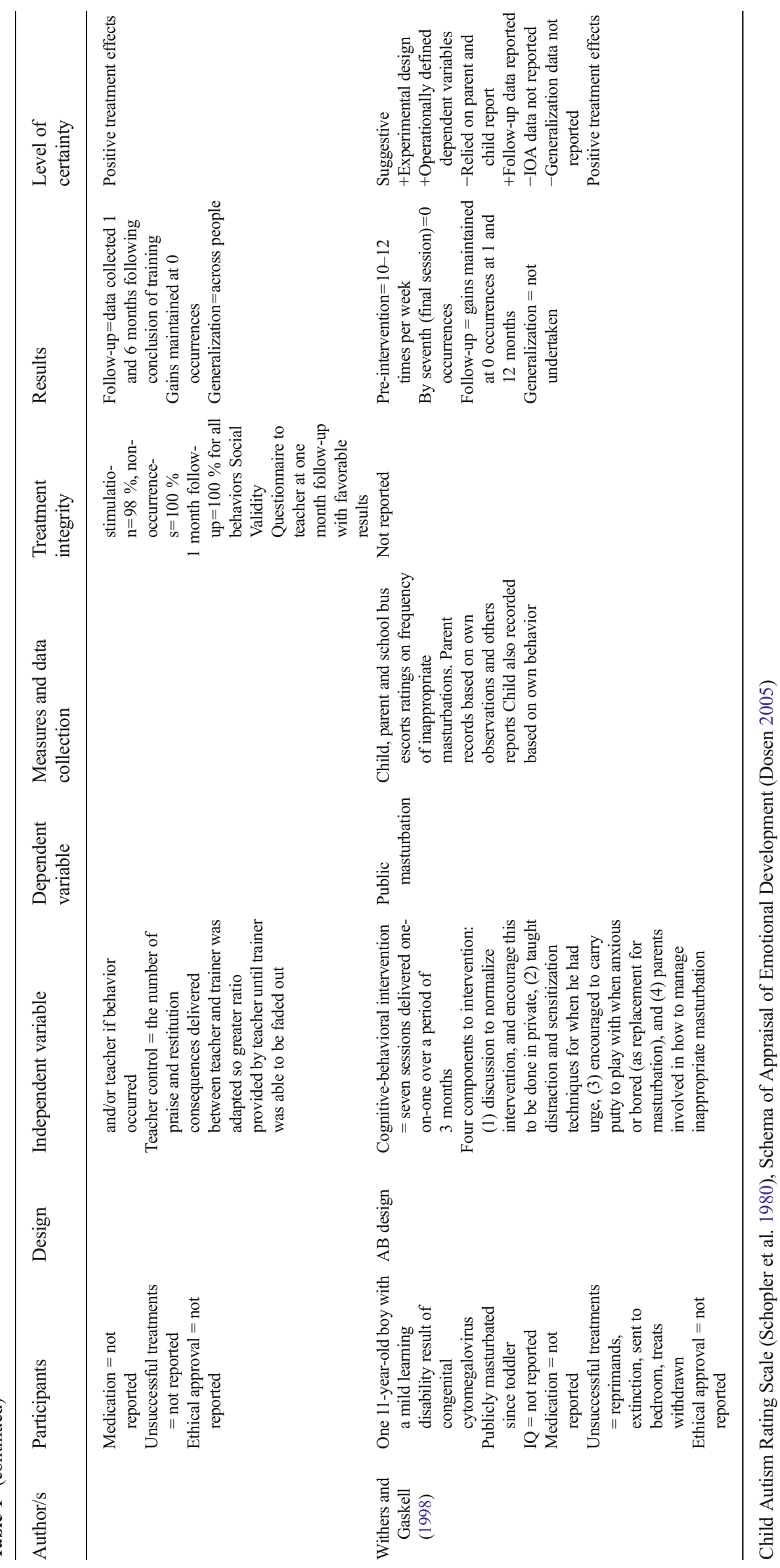


this an accurate summary of the participants? (b) is this an accurate report of the dependent variables? (c) is this an accurate report of the experimental design? (d) is this an accurate description of the data collection measures and procedures? (e) is this an accurate report of the independent variables? (f) is this an accurate summary of treatment integrity data? $(\mathrm{g})$ is this an accurate summary of the results of the study? (h) is this an accurate reflection of the certainty of evidence? There were 96 possible items on which there could be agreement or disagreement (i.e., eight items for each of the 12 studies). There was $95.8 \%$ agreement between the first and second authors for each of the items that were coded. Any discrepancies between the first and second authors were resolved through further review of each paper and discussion of differences until $100 \%$ agreement was reached.

\section{Results}

\section{Participants}

Collectively, a total of 50 participants were included across the 12 studies. Butler and Fontenelle-III (1995) conducted a between groups study which included 30 participants. Coskun et al. (2009) conducted a single-case AB design study that included 10 participants. The remaining 10 studies included one participant each. The participants in the 12 studies consisted of three females and 47 males and ranged between 5 and 16 years of age.

Twelve of the 50 participants included in this review had a diagnosis of ASD (Albertini et al. 2006; Coskun and Mukaddes 2008; Coskun et al. 2009), one child had moderate spastic hemiplegia, microcephaly, and severe mental retardation (Cook et al. 1978), one child had fixed encephalopathy and severe psychomotor retardation (Czyzewski et al. 1982), one child had a traumatic brain injury and seizure disorder (Fyffe et al. 2004), one child had Down syndrome (Polvinale and Lutzker 1980), and one child had a mild learning disability (Withers and Gaskell 1998). In the remaining studies, participants were simply described as having a level of intellectual disability, but further diagnostic information was not provided.

The IQ of the participants was reported in five of the 12 studies (Albertini et al. 2006; Barmann and Murray 1981; Butler and Fontenelle-III 1995; Foxx et al. 1986; Polvinale and Lutzker 1980). IQ scores ranged from severe intellectual disability to being in the normal range for one of the participants with ASD. As so few studies reported the IQ of the participants, it is difficult to draw any conclusions regarding potential relationships between IQ, ISB, and response to treatment.

Three of the studies included participants who were on medication to treat co-existing conditions and/or behaviors.
This included seizure medications (Cook et al. 1978; Coskun and Mukaddes 2008), risperidone (Coskun and Mukaddes 2008; Coskun et al. 2009), and olanzapine (Coskun et al. 2009). These medications were not specified as being used to treat ISB.

Four studies reported unsuccessful use of other interventions prior to the reported treatments. Barmann and Murray (1981) reported previous treatments such as differential reinforcement of other behaviors (DRO), verbal reprimands, extinction, time out, and overcorrection procedures. Foxx et al. (1986) had unsuccessfully used teacher reprimands, scolding, and differential reinforcement of incompatible behaviors (DRI) in the form of hands on table. Luiselli et al. (1977) had attempted stimulus control procedures, while Withers and Gaskell (1998) tried reprimands, extinction, sending the child to the bedroom, and the withdrawal of treats. It is also noteworthy that two of the studies reported that the participants had a history of several years of engaging in ISB (Polvinale and Lutzker 1980; Withers and Gaskell 1998).

\section{Experimental Design}

Six of the 12 studies included in this review used a single-case experimental design (Barmann and Murray 1981; Cook et al. 1978; Czyzewski et al. 1982; Foxx et al. 1986; Fyffe et al. 2004; Polvinale and Lutzker 1980). Two of these studies included a multiple baseline across settings (Barmann and Murray 1981; Cook et al. 1978), one across time of day (Foxx et al. 1986), and one across settings and time of day (Polvinale and Lutzker 1980). Two of these studies used an ABAB reversal design (Czyzewski et al. 1982; Fyffe et al. 2004). The remaining studies used a simple $A B$ design (Coskun et al. 2009; Luiselli et al. 1977; Withers and Gaskell 1998) or case report format (Albertini et al. 2006; Coskun and Mukaddes 2008). Only one of the studies employed a between groups design (Butler and FontenelleIII 1995).

\section{Independent Variables}

Various intervention strategies were used to treat ISB. The majority of the 12 studies that used behavioral strategies used a multi-component intervention approach. Procedures included overcorrection (Czyzewski et al. 1982; Luiselli et al. 1977), social restitution (Polvinale and Lutzker 1980), positive practice (i.e., the child was asked to perform another motor task) (Czyzewski et al. 1982), the use of differential reinforcement including DRO (Foxx et al. 1986; Polvinale and Lutzker 1980), DRI (Luiselli et al. 1977), as well as the use of reinforcement for the absence of the ISB (Czyzewski et al. 1982; Foxx et al. 1986). Two studies also used extinction procedures (e.g., ignoring the ISB) as a component of their intervention package (Fyffe et al. 2004; Luiselli et al. 1977). The Fyffe 
et al. (2004) study was unique in that the authors incorporated functional communication training as a component of intervention where the child was required to hand over a card in order to request attention from others.

Two studies relied solely on the use of aversive procedures: one in the form of facial screening paired with negative feedback contingent upon the display of ISB (Barmann and Murray 1981), and the other aversive involved squirting lemon juice into the child's mouth contingent upon public masturbation (Cook et al. 1978). It is noteworthy that following intervention, the participant in the Barmann and Murray (1981) study participated in a sex education program designed to teach people with disabilities to appropriately express their sexuality.

Three of the studies identified used a pharmacological approach for the treatment of inappropriate sexual behavior. This included the use of antidepressant medications, which can reportedly result in sexual dysfunction (Gregorian et al. 2002). The medication prescribed in these three studies was mirtazapine (Albertini et al. 2006; Coskun and Mukaddes 2008; Coskun et al. 2009). Mirtazapine is a noradrenergic and specific serotonergic antidepressant (NaSSA) that has been used effectively in the treatment of clinical depression among adults and the elderly (Croom et al. 2008). There is also a tentative suggestion that mirtazapine may reduce some of the symptoms of ASD and other pervasive developmental disorders (Posey et al. 2001). Coskun et al. (2009) selected the use of mirtazapine as it was reported to have potential antilibidal effects.

One of the 12 studies identified used a cognitive-behavioral intervention (Withers and Gaskell 1998). This consisted of (1) discussion with the participant in order to normalize intervention and encourage them to engage in sexual behavior in private, (2) teaching distraction and sensitisation techniques for when he had a sexual urge, (3) encouraging the participant to carry putty to play with when anxious or bored (as replacement for masturbation), and (4) parent education in how to manage inappropriate masturbation.

The research conducted by Butler and Fontenelle-III (1995) used a group therapy approach. In this study, the 30 participants were assigned to one of three treatment groups. The sexual intervention group received eight group therapy sessions which focused on strategies to reduce ISB including facilitated group discussion regarding appropriate sexual conduct, modeling of appropriate social and sexual behaviors, role play, and then feedback based on real-world interactions. The behavioral intervention group was also provided eight group sessions but focused on inappropriate behaviors, without specifying a specific target behavior. This included discussion of inappropriate behaviors (excluding specific reference to sexual behavior), role play and modeling of provocative scenarios, and real-world interaction combined with group and non-group members. It is noteworthy that inappropriate sexual behaviors were only raised and discussed once by this group. The third group consisted of a no treatment control group.

\section{Dependent Variables}

Public masturbation was identified as a focus of treatment in nine of the 12 studies (Albertini et al. 2006; Barmann and Murray 1981; Butler and Fontenelle-III 1995; Cook et al. 1978; Coskun et al. 2009; Czyzewski et al. 1982; Foxx et al. 1986; Luiselli et al. 1977; Withers and Gaskell 1998). In one of these studies, this also included the public display of genitalia (Barmann and Murray 1981). In three of the identified studies, one of the target behaviors included the inappropriate and uninvited sexual touching of another person (Butler and Fontenelle-III 1995; Coskun et al. 2009; Fyffe et al. 2004). In the two studies that provided group intervention, several target behaviors were identified. For example, in the Butler and Fontenelle-III (1995) study, the 30 participants who were the focus of intervention engaged in what was referred to as frequent and recurrent sexual acting out behavior. These behaviors included uninvited and inappropriate sexual touching of another person, public self-stimulatory behavior, exhibitionist behavior, and the inappropriate and public use of sexual language. In the Coskun et al. (2009) study, the target behaviors included public masturbation, inappropriate touching of others, public disrobing, arousal resulting from specific body parts or inanimate objects, and observation of others disrobing or bathing.

The Coskun and Mukaddes (2008) study was unique in that the participant demonstrated fetishistic behavior in which they would become aroused by another person wearing blue jeans. This would result in the child making attempts to rub their genitalia against that person.

Polvinale and Lutzker (1980) focused on three categories of behavior which they labelled (1) assaultive behavior (nonsexual), (2) inappropriate interpersonal sexual behavior, and (3) genital self-stimulation. No further definition of these behaviors was provided.

\section{Measures and Data Collection Procedures}

The majority of studies used direct observation techniques to record the frequency of ISB (Barmann and Murray 1981; Butler and Fontenelle-III 1995; Cook et al. 1978; Czyzewski et al. 1982; Foxx et al. 1986; Fyffe et al. 2004; Luiselli et al. 1977; Polvinale and Lutzker 1980; Withers and Gaskell 1998). Time sample, fixed-interval frequency recordings were used in several of the studies to measure changes in ISB (Barmann and Murray 1981; Cook et al. 1978; Czyzewski et al. 1982; Foxx et al. 1986; Fyffe et al. 2004; Luiselli et al. 1977; Polvinale and Lutzker 1980) during baseline, intervention, and with the exception of Fyffe et al. (2004) 
during post-intervention or follow-up. Some studies recorded the frequency of ISB as it occurred throughout the day (Butler and Fontenelle-III 1995; Withers and Gaskell 1998). Coskun and Mukaddes (2008) and Coskun et al. (2009) reported that they encouraged parents to monitor the frequency and the duration of the ISB. However, this data was not presented in the results, and there was insufficient detail to allow for replication of these procedures. The Coskun et al. (2009) study was unique in that it also incorporated video observation in order to determine the characteristics, severity, frequency, duration, and impact of ISB on the participants' adaptive functioning. The Withers and Gaskell (1998) study was also novel as it included child-reported change in addition to parentreported change.

Several standardized norm-referenced measures were used for general changes in behavior in some of the studies reviewed. For example, Albertini et al. (2006) administered the Child Autism Rating Scale (CARS) (Schopler et al. 1980) and Schema of Appraisal of Emotional Development (Dosen 2005) during the first clinical evaluation and then 6 months following treatment. Coskun et al. (2009) administered the Clinical Global Impressions-Severity (CGI-S) scale and Clinical Global Impressions-Improvement (CGI-I) scale at baseline and at the conclusion of their study in order to measure changes in ISB.

\section{Treatment Integrity}

Treatment integrity data was not reported in six of the 12 studies. Those that did not report treatment integrity data were Albertini et al. (2006), Butler and Fontenelle-III (1995), Coskun and Mukaddes (2008), Coskun et al. (2009), Czyzewski et al. (1982), and Withers and Gaskell (1998). Six of the studies collected IOA data over a proportion of phases (Barmann and Murray 1981; Cook et al. 1978; Foxx et al. 1986; Fyffe et al. 2004; Luiselli et al. 1977; Polvinale and Lutzker 1980). One of the studies reported on the procedures that were used to train those implementing intervention procedures (Barmann and Murray 1981), while another reported the data recording procedures (Czyzewski et al. 1982). Only Polvinale and Lutzker (1980) described social validity outcomes.

\section{Results}

Eleven of the 12 studies reported positive treatment effects with one study reporting mixed treatment effects (Butler and Fontenelle-III 1995). Those approaches which incorporated overcorrection (Czyzewski et al. 1982; Luiselli et al. 1977) and reinforcement procedures such as DRO (Foxx et al. 1986) as a component of the intervention package reported a reduction in masturbation behavior. Luiselli et al. (1977) reported complete elimination of this behavior. Interestingly, in the Polvinale and Lutzker (1980) study, the use of DRO alone only resulted in a slight reduction in ISB. The introduction of DRO combined with social restitution, however, resulted in the elimination of all three target behaviors. Similarly, Fyffe et al. (2004) found that the teaching of functional communication training resulted in a $94 \%$ reduction in ISB.

Of the two studies which used aversive procedures in response to ISB (Barmann and Murray 1981; Cook et al. 1978), a decrease in ISB from baseline recordings was reported; thus, treatment effects were considered to be positive. In one of these studies (Cook et al. 1978), ISB ceased to occur following treatment, and in the Barmann and Murray (1981) study, a $98 \%$ decrease in self-stimulatory behavior in the classroom, a $91 \%$ decrease on the school bus, and a $92 \%$ decrease in the home were reported.

Of the three studies that used the mirtazapine treatment, the effects were reported to be positive. Two studies reported that the ISB ceased to exist following treatment (Albertini et al. 2006; Coskun and Mukaddes 2008). For the remaining study (Coskun et al. 2009), a significant difference between the CGI-S scores between baseline and following intervention was reported. Five of the nine participants who completed the study were reported to demonstrate "very much improvement," three showed "much improvement," and one showed "moderate improvement" in excessive masturbation. Six out of the 10 participants were also reported to have demonstrated improvement in other ISB.

In the one study that used a group therapy approach (Butler and Fontenelle-III 1995), the effects of treatment were mixed. Data analysis revealed a significant reduction in sexual acting out behavior between pre- and post-test scores for the sexual intervention group. It was also evident that those in the sexual intervention group showed the most significant reduction in the target behavior when compared to the behavioral intervention and control groups. In the sole study that used an individual cognitive-behavioral intervention (Withers and Gaskell 1998), masturbation behavior decreased from 10-12 times per week prior to intervention to an absence of the behavior by the seventh and final treatment session.

As 10 of the 12 studies included only one participant in their research design, few statistical analyses of findings were conducted. Butler and Fontenelle-III (1995) conducted an ANOVA to compare gains made between pre-test and posttest scores across treatment groups. Coskun et al. (2009) conducted Wilcoxon nonparametric $t$ tests to measure the changes in CGI-S scores between baseline and post-treatment.

\section{Follow-up and Generalization}

Generalization data was only reported for three of the 12 studies included in this review (Cook et al. 1978; Foxx et al. 1986; Polvinale and Lutzker 1980). Foxx et al. (1986) measured the transfer of learned behavior to school staff when these staff 
commenced treatment procedures. The authors found that treatment effects were maintained when staff were responsible for implementing treatment procedures. In the Cook et al. (1978) study, treatment was initially implemented in the school setting, followed by the home setting. Probes for generalization to the home setting were conducted when parents began implementing the study protocol. Finally, Polvinale and Lutzker (1980) conducted behavior probes to determine whether treatment effects were maintained when the trainer was not present. These gains were maintained in the school setting in the absence of the trainer.

Seven of the studies in this review included measures to assess the maintenance of treatment effects (Barmann and Murray 1981; Cook et al. 1978; Czyzewski et al. 1982; Foxx et al. 1986; Luiselli et al. 1977; Polvinale and Lutzker 1980; Withers and Gaskell 1998). This ranged from 2 weeks (Foxx et al. 1986) to 12 months post-treatment (Luiselli et al. 1977; Withers and Gaskell 1998). In each of these seven studies, the treatment effects were maintained following a period without intervention (Barmann and Murray 1981; Cook et al. 1978; Czyzewski et al. 1982; Foxx et al. 1986; Luiselli et al. 1977; Polvinale and Lutzker 1980; Withers and Gaskell 1998). Albertini et al. (2006), Coskun and Mukaddes (2008), Coskun et al. (2009), and Fyffe et al. (2004) did not report follow-up data.

\section{Certainty of Evidence}

In each study, the certainty of evidence was categorized according to whether it was suggestive, preponderant, or conclusive. For eight studies, the certainty of evidence was suggestive (Albertini et al. 2006; Butler and Fontenelle-III 1995; Coskun and Mukaddes 2008; Coskun et al. 2009; Czyzewski et al. 1982; Fyffe et al. 2004; Luiselli et al. 1977; Withers and Gaskell 1998). The certainty of evidence was preponderant for three studies (Cook et al. 1978; Foxx et al. 1986; Polvinale and Lutzker 1980). The certainty of evidence was considered to be conclusive for only one study (Barmann and Murray 1981). As identified in Table 1, articles which were classified as having a suggestive level of certainty for intervention effects generally used an AB experimental design, did not collect follow-up or generalization data, did not include measures of treatment integrity, did not operationally define the target behavior, and/or did not include sufficient detail of the data recording procedures to allow replication. By contrast, the studies that provided a preponderant or conclusive level of certainty met all of the aforementioned criteria. Foxx et al. (1986) only provided replication across settings on one occasion, Polvinale and Lutzker (1980) did not sufficiently control for the effects of extraneous variables, and Cook et al. (1978) included only a small percentage of IOA data which meant that the results of these studies were unable to be classified as providing conclusive evidence of treatment effects. The distinguishing feature of the study that provided a conclusive level of certainty is that it is thought to have included sufficient methodological rigor to rule out alternative explanations for treatment effects (Barmann and Murray 1981).

In summary, 11 of the studies reviewed demonstrated positive treatment effects. However, it is possible that some components of intervention when used in isolation (e.g., DRO) may not produce positive treatment outcomes (Polvinale and Lutzker 1980). The findings of these studies should also be interpreted in light of their procedural limitations.

\section{Discussion}

This systematic review identified 12 studies published between 1977 to 2009 that had examined the effectiveness of treatments for ISB in children and adolescents with developmental disabilities. The treatments for ISB included pharmacological and behavioral interventions, and each reported some positive treatment outcomes in the form of a decrease or elimination of ISB. Given the paucity of studies in this area and in conjunction with the limited number of participants in each study, the authors of this review are restricted in terms of the overall conclusions that are able to be drawn regarding potentially efficacious treatments for ISB.

A number of limitations were identified in the research that warrants further discussion. Only one of the studies reached a conclusive level of certainty, with the majority of research studies identified as having a suggestive level of certainty. One of the primary reasons for this is due to the experimental design utilized in each of the studies. Several of the studies that were included in this review used a single-case $A B$ research design (Coskun and Mukaddes 2008; Coskun et al. 2009; Luiselli et al. 1977; Withers and Gaskell 1998). This was the case for each of those studies that investigated the use of mirtazapine as a treatment for ISB (Albertini et al. 2006; Coskun and Mukaddes 2008; Coskun et al. 2009) and also the study that used cognitive-behavioral intervention (Withers and Gaskell 1998). While it is acknowledged that it can be unethical to implement a reversal design for behaviors of this nature, the use of an $\mathrm{AB}$ design in addition to other procedural limitations means that it is difficult to conclude that the positive treatment effects reported in these studies occurred as a result of intervention.

In addition to the limitations associated with study design, the small number of participants that are included in each study threatens the external validity of the findings and makes it difficult to generalize the study outcomes across age, gender, disability, IQ, and sexual behaviors. While some of the research studies were strengthened by the use of a multiple baseline design, 10 of the 12 studies included only a single participant. 
Some of the studies also presented with issues that may have affected measurement validity (Albertini et al. 2006; Coskun and Mukaddes 2008; Coskun et al. 2009; Withers and Gaskell 1998). For example, some of the outcome measures used predominantly relied upon parent- or childreported changes in behavior (Coskun and Mukaddes 2008; Withers and Gaskell 1998), direct recording of the ISB was often not conducted (Albertini et al. 2006; Coskun and Mukaddes 2008; Coskun et al. 2009), and in some cases the specific changes in ISB data were not presented (Albertini et al. 2006; Coskun and Mukaddes 2008). The potential for bias associated with use of self-report or parent report rather than direct observation of the behavior may have impacted on the reliability of the findings that were reported. The issues associated with measurement validity may be further confounded by the fact that dependent variables were not always operationally defined (Albertini et al. 2006; Coskun and Mukaddes 2008). As a result, the replicability of these studies is compromised.

It is noteworthy that many of those studies which provided behavioral treatment used time sample and frequency recordings in which IOA data were also gathered (Barmann and Murray 1981; Cook et al. 1978; Foxx et al. 1986; Fyffe et al. 2004; Luiselli et al. 1977; Polvinale and Lutzker 1980). As a result, these studies approached a preponderant or conclusive level of certainty and therefore showed promise as effective approaches to intervention for ISB.

\section{Clinical Implications}

There are several key implications that should be considered when interpreting these findings. Firstly, it appears that the use of a multi-component behavioral intervention can aide in the reduction or elimination of ISB. This is a finding that has been demonstrated not only with disabled children and adolescents with ISB but also with adults with an intellectual disability (Le Blanc et al. 2000). While it is difficult to isolate which components of the intervention were effective, it appears that the use of differential reinforcement procedures and the teaching of appropriate replacement skills or functional alternatives (e.g., through functional communication training) are an important component of intervention for ISB. Equally, while the use of aversive procedures in the form used in this review would no longer be considered ethical, it is possible that overcorrection and social restitution procedures, contingent upon the occurrence of the ISB, may be an effective component of the treatment protocol. Given this possibility, a complete functional assessment of the ISB might be warranted in the development of an intervention plan. Also, despite limitations associated with the study design, the use of mirtazapine as a treatment for ISB may have potential given the positive report of treatment effects. Finally, based on the findings of Butler and Fontenelle-III (1995), it seems important that when used in group intervention, a cognitive-behavior intervention that specifically targets ISB and awareness of sexual issues, as opposed to a more general behavioral focus, may result in greater compliance and responsiveness to the target goals. These findings each require replication in order to substantiate the evidence of potentially positive treatment effects.

It is noted that in spite of an ideological shift in the way in which the sexual behavior of people with developmental disabilities is viewed, the approach to treatment of ISB between 1977 and 2009 remains relatively unchanged. The majority of treatment over this time period has been based on the principles of applied behavior analysis. The exception being three recent studies which have used medication as the treatment of choice (Albertini et al. 2006; Coskun and Mukaddes 2008; Coskun et al. 2009). The use of medication as a treatment for ISB needs to be considered carefully. Using medication without also teaching children and adolescents strategies to manage ISB may mean that medications are unable to be faded and therefore may not lead to sustained change in ISB.

In addition to direct clinical implications, it is very important to consider the home and community impact of learning to refrain from ISB for children and adolescents with developmental disabilities. Given the way in which ISB can impact upon community access and engagement, social, familial, and intimate relationships, and school inclusion, it is essential to design and develop interventions for ISB for use across multiple settings or in which outcomes generalize across relevant settings. For example, intervention may focus on teaching functional alternatives to the ISB that can be applied in multiple contexts and across multiple people.

As a final point, very few studies included female participants. Only three of the 50 participants collectively were female. It is recognized that there can be gender differences in the patterns of sexual behavior that are exhibited, though there is little research which describes the differences in the ways in which ISB is demonstrated in males and females. There are some suggestions, for typically developing children, that social and cultural factors and also anatomical differences may lead to referral biases in which males are referred more frequently for treatment than females (Mallants and Casteels 2008; Yang et al. 2005). The lack of research in this area makes it very difficult to draw conclusions about any gender differences in terms of the expression of sexual acting out behavior, the severity of ISB, and response to treatment. Furthermore, it raises the question of whether there are gender differences in terms of the rates and/or the manifestation or demonstration of ISB between males and females.

\section{Future Research}

It is essential that a better understanding of the ways in which treatment of ISB in children and adolescents with developmental disabilities be developed. This commonly occurring 
behavior can impact profoundly on children's and adolescents' social, familial and intimate relationships, participation and inclusion in school, and integration and functioning in the wider community. Given there has been an ideological shift away from viewing the sexual behavior of people with disabilities as deviant or simply absent, it is imperative that support is given to these children and young people so that they can appropriately express and manage their sexual behavior. In order to do this and as identified in this review, there are several key areas that require further exploration and supported by the findings of others in this field (Lockhart et al. 2009). Primarily, further replication of the treatment procedures used in this review would help to validate these approaches. This includes the use of both pharmacological and behavioral approaches, as well as cognitive-behavioral interventions delivered one-on-one and potentially, in a group therapy format. Cognitive-behavioral interventions are often delivered in a group therapy format, and therefore, it is important that we understand whether this is an efficacious approach for this population. It would also be interesting to conduct further investigation into treatment for types of ISB other than public masturbation (e.g., fetishistic behavior) because, to date, there is paucity of research that has focused on this spectrum of ISB. Finally, Mallants and Casteels (2008) recommended parent and child education as a viable treatment for childhood masturbation. There appears to be little research implementing educational procedures with children and adolescents with disabilities. The exception to this is the studies by Butler and Fontenelle-III (1995) and Withers and Gaskell (1998) that demonstrated the potential benefits of sex education when provided to adolescents. Further exploration into the effectiveness of this approach is certainly warranted given that it serves an educational purpose and is a non-aversive treatment option.

While a small number of the 12 research studies employed a sound methodology, there are several important considerations that could be made in the design of future research. In order to increase our understanding of the efficacy of certain treatment approaches, it is important that further research includes replication across a greater number of participants. In particular, it would be valuable to identify any gender differences in the types of ISB exhibited and any variation in response to treatment between males and females. There is a need for more rigorous, experimentally designed studies that include multiple baseline designs or in the case of pharmacological treatments the need for randomized controlled trial studies. In order to maintain the social validity of the research in this area, it is important that studies consistently assess the generalization of treatment outcomes across settings and people. It is equally important to ensure that procedures are put in place to measure the maintenance of treatment effects. There is also a need to provide clearly defined, replicable data recording procedures, which include direct observation of ISB. This data should include measures of treatment integrity (e.g.,
IOA reliability data). Finally, as many of the treatments implemented were multi-component interventions, it would be interesting to isolate which features of intervention contribute towards treatment outcomes. For example, in the Polvinale and Lutzker (1980) study, intervention was not as effective until social restitution procedures were implemented suggesting that this may be a fundamental component of the treatment process. Monitoring these procedural variables will ensure that the positive treatment effects that are reported are not accounted for by other extraneous variables.

\section{Conclusion}

In recent years, there has been an ideological shift away from viewing the sexual behavior of individuals with disabilities as pathological or deviant. In spite of this change in thinking, it remains that many individuals with disabilities have difficulty expressing their sexual behavior in an appropriate time, place, and manner. To date, there is a paucity of research that has investigated the efficacy of treatments for ISB in children and adolescents with developmental disabilities. It is essential that further methodologically sound research is conducted in order to enhance our understanding of evidence-based treatments for ISB. Providing effective treatment for this behavior has many important implications for social, developmental, community integration, and educational outcomes for this population.

Conflict of Interest The authors declare that they have no competing interests.

\section{References}

Albertini, G., Polito, E., Sara, M., Di Gennaro, G., \& Onorati, P. (2006). Compulsive masturbation in infantile autism treated by mirtazapine. Pediatric Neurology, 34, 417-418.

Barmann, B. C., \& Murray, W. J. (1981). Suppression of inappropriate sexual behavior by facial screening. Behavior Therapy, 12, 730735.

Barnhill, G. P. (2007). Outcomes in adults with Asperger syndrome. Focus on Autism and Other Developmental Disabilities, 22, 116126.

Baron-Cohen, S. (2001). Theory of mind in normal development and autism. Prisme, 34, 174-183.

Butler, S. F., \& Fontenelle-III, S. F. (1995). Cognitive-behavioral group therapy: application with adolescents who are cognitively impaired and sexually act out. The Journal for Specialists in Group Work, 20, $121-127$

Clements, J., \& Zarowska, E. (2000). Behavioral concerns and autistic spectrum disorder: explanations and strategies for change. London: Jessica Kingsley.

Cook, J. W., Altman, K., Shaw, J., \& Blaylock, M. (1978). Use of contingent lemon juice to eliminate public masturbation by a severely retarded boy. Behaviour Research and Therapy, 16, 131-134. 
Coskun, M., \& Mukaddes, N. M. (2008). Mirtazapine treatment in a subject with autistic disorder and fetishism. Journal of Child and Adolescent Psychopharmacology, 18, 206-209.

Coskun, M., Karakoc, S., Kircelli, F., \& Mukaddes, N. M. (2009). Effectiveness of mirtazapine in the treatment of inappropriate sexual behaviors in individuals with autistic disorder. Journal of Child and Adolescent Psychopharmacology, 19, 203-206.

Croom, K. F., Perry, C. M., \& Plosker, G. L. (2008). Mirtazapine: a review of its use in major depression and other psychiatric disorders. CNS Drugs, 23, 427-452.

Czyzewski, M. J., Barrera, R. D., \& Sulzer-Azaroff, B. (1982). An abbreviated overcorrection program to reduce self-stimulatory behaviors. Journal of Behavior Therapy and Experimental Psychiatry, 13, 55-62.

Dalldorf, J. (1983). Medical needs of the autistic adolescent. In E. Schopler \& G. Mesibov (Eds.), Autism in adolescents and adults and adults (pp. 149-168). New York: Plenum Press.

Dosen, A. (2005). Applying the developmental perspective in the psychiatric assessment and diagnosis of persons with intellectual disability: part I-assessment; part II-diagnosis. Journal of Intellectual Disability Research, 49, 1-15.

Early, M. C., Erickson, C. A., \& Wink, L. K. (2012). Case report: 16-yearold male with autistic disorder with preoccupation with female feet. Journal of Autism and Developmental Disorders, 42, 1133-1137.

Eaves, L. C., \& Ho, H. H. (1996). Stability and change in cognitive and behavioral characteristics of autism through childhood. Journal of Autism and Developmental Disability, 26, 557-569.

Foxx, R. M., McMorrow, M. J., Fenlon, S., \& Bittle, R. G. (1986). The reductive effects of reinforcement procedures on the genital stimulation and stereotypy of a mentally retarded adolescent male. Analysis and Intervention in Developmental Disabilities, 6, 239-248.

Friedrich, W. N., Fisher, J., Broughton, D., Houston, M., \& Shafran, C. R. (1998). Normative sexual behavior in children: a contemporary sample. Pediatrics, 101, E9. doi:10.1542/peds.101.4.e9.

Fyffe, C. E., Kahng, S. W., Fittro, E., \& Russell, D. (2004). Functional analysis and treatment of inappropriate sexual behaviour. Journal of Applied Behavior Analysis, 37, 401-404.

Gerhardt, P. (2006). Sexuality instruction and autism spectrum disorders. Autism \& Asperger's Digest Magazine, 44-46.

Gougeon, N. A. (2010). Sexuality and autism: a critical review of selected literature using a social-relational model of disability. American Journal of Sexuality Education, 5, 328-361.

Gregorian Jr, R. S., Golden, K. A., Bahce, A., Goodman, C., Kwong, J. W., \& Kahn, Z. M. (2002). Antidepressant induced sexual dysfunction. Annals of Pharmacotherapy, 36, 1577-1589.

Hatton, S., \& Tector, A. (2010). Sexuality and relationship education for young people with autistic spectrum disorder: curriculum change and staff support. British Journal of Special Education, 37, 69-76.

Hellemans, H., Colson, K., Verbraeken, C., Vermeiran, R., \& Deboutte, D. (2007). Sexual behavior in high-functioning male adolescents and young adults with autism spectrum disorder. Journal of Autism and Developmental Disorders, 37, 260-269.

Jones, L., Bellis, M. A., Wood, S., Hughes, K., McCoy, E., Eckley, L., Bates, G., Mikton, C., Shakespeare, T., \& Officer, A. (2012). Prevalence and risk of violence against children with disabilities: a systematic review and meta-analysis of observational studies. Lancet, 380, 899-907.

Koller, R. (2000). Sexuality and adolescents with autism. Sexuality and Disability, 18, 125-135.

Le Blanc, L. A., Hagopian, L. P., \& Maglieri, K. A. (2000). Use of a token economy to eliminate excessive inappropriate social behavior in an adult with developmental disabilities. Behavioral Interventions, 15, 135-143.

Leung, A. K. C., Robson, W. L. M., \& Tay-Uyboco, J. (1993). The incidence of labial fusion in children. Journal of Paediatrics and Child Health, 29, 235-236.

Lockhart, K., Guerin, S., Shanahan, S., \& Coyle, K. (2009). Defining "sexualized challenging behavior" in adults with intellectual disabilities. Journal of Policy and Practice in Intellectual Disabilities, 6, 293-301.
Ludlow, B. L. (1991). Contemporary issues in sexuality and mental retardation. Advances in Mental Retardation and Developmental Disabilities, 4, 1-26.

Luiselli, J. K., Helfen, C. S., Pemberton, B. W., \& Reisman, J. (1977). The elimination of a child's in-class masturbation by overcorrection and reinforcement. Journal of Behavior Therapy and Experimental Psychiatry, 8, 201-204.

Mallants, C., \& Casteels, K. (2008). Practical approach to childhood masturbation: a review. European Journal of Pediatrics, 167, 1111-1117.

Mallika, B., Pragna, R., \& Amrita, P. (2013). Role of sex education on odd sexual and problem behavior a study on adolescents with autism. Indian Journal of Community Psychology, 9, 1-20.

Meizner, I. (1987). Sonographic observation of in utero fetal "masturbation". Journal of Ultrasound Medicine, 6, 111.

Milligan, M. S., \& Neufeldt, A. H. (2001). The myth of asexuality: a survey of social and empirical evidence. Sexuality and Disability, 19, 91-109.

Motzin, B. (1993). Precocious puberty: diagnosis, evaluation and management. Pediatrics in Review, 14(33), 336-367.

Mulloy, A., Lang, R., O’Reilly, M., Sigafoos, J., Lancioni, G., \& Rispoli, M. (2010). Gluten-free and casein-free diets in the treatment of autism spectrum disorders: a systematic review. Research in Autism Spectrum Disorders, 4, 328-339.

Polvinale, R. A., \& Lutzker, J. R. (1980). Elimination of assaultive and inappropriate sexual behavior by reinforcement and social restitution. Mental Retardation, 18, 27-30.

Posey, D. J., Guenin, K. D., Kohn, A. E., Swiezy, N. B., \& McDougie, C. J. (2001). A naturalistic open-label study of mirtazapine in autistic and other pervasive developmental disorders. Journal of Child and Adolescent Psychopharmacology, 11, 267-277.

Realmuto, G. M., \& Ruble, L. A. (1999). Sexual behaviors in autism: problems of definition and management. Journal of Autism and Developmental Disorders, 29, 121-127.

Ruble, L., \& Dalrymple, N. (1993). Social/sexual awareness of persons with autism: a parental perspective. Archives of Sexual Behavior, 22, 229-240.

Sandfort, T. G. M., \& Rademakers, J. (2000). Childhood sexual development: normal sexual behavior and development. Binghamton: The Haworth Press Inc.

Schaafsma, D., Stoffelen, J. M. T., Kok, G., \& Curfs, L. M. G. (2013). Exploring the development of existing sex education programmes for people with intellectual disabilities: an intervention mapping approach. Journal of Applied Research in Intellectual Disabilities, $26,157-166$

Schopler, E., Reichler, R. J., DeVellis, R. F., \& Daly, K. (1980). Toward objective classification of childhood autism: Childhood Autism Rating Scale (CARS). Journal of Autism and Developmental Disorders, 10, 91-103.

Simeonson, R., \& Bailey, D. (1991). Evaluating programme impact: levels of certainty. In D. Mitchell \& R. Brown (Eds.), Early intervention studies for young children with special needs. New York: Chapman and Hall.

Smith, N. (1981). The certainty of evidence in health evaluations. Evaluation and Program Planning, 4, 273-278.

Stokes, T. F., \& Baer, D. M. (1977). An implicit technology of generalization. Journal of Applied Behavior Analysis, 10, 349-367.

Stokes, M. A., \& Kaur, A. (2005). High-functioning autism and sexuality: a parental perspective. Autism, 9, 266-289.

Sullivan, A., \& Caterino, L. C. (2008). Addressing the sexuality and sex education of individuals with autism spectrum disorder. Education and Treatment of Children, 31, 381-394.

Tarnai, B. (2006). Review of effective interventions for socially inappropriate masturbation in persons with cognitive disabilities. Sexuality and Disability, 24, 151-168. 
Withers, P. S., \& Gaskell, S. L. (1998). A cognitive-behavioural intervention to address inappropriate masturbation in a boy with mild learning disabilities. British Journal of Learning Disabilities, 26, 58-61.
Yang, M. L., Fullwood, E., Goldstein, J., \& Mink, J. W. (2005). Masturbation in infancy and early childhood presenting as a movement disorder: 12 cases and a review of the literature. Pediatrics, $116,1427-1432$. 